\title{
POSITIVE SOLUTIONS TO $p(x)$-LAPLACIAN-DIRICHLET PROBLEMS WITH SIGN-CHANGING NON-LINEARITIES
}

\author{
XIANLING FAN \\ Department of Mathematics, Lanzhou City University, Lanzhou 730070, PR China \\ Department of Mathematics, Lanzhou University, Lanzhou 730000, PR China \\ e-mail:fanxl@lzu.edu.cn
}

(Received 8 March 2009; revised 14 January 2010; accepted 21 February 2010)

\begin{abstract}
Consider the $p(x)$-Laplacian-Dirichlet problem with sign-changing non-linearity of the form$$
\begin{cases}-\operatorname{div}\left(|\nabla u|^{p(x)-2} \nabla u\right)+m(x)|u|^{p(x)-2} u=\lambda a(x) f(u) & \text { in } \Omega \\ u=0 & \text { on } \partial \Omega\end{cases}
$$

where $\Omega \subset \mathbb{R}^{N}$ is a bounded domain, $p \in C^{0}(\bar{\Omega})$ and $\inf _{x \in \bar{\Omega}} p(x)>1, m \in L^{\infty}(\Omega)$ is non-negative, $f: \mathbb{R} \rightarrow \mathbb{R}$ is continuous and $f(0)>0$, the coefficient $a \in L^{\infty}(\Omega)$ is sign-changing in $\Omega$. We give some sufficient conditions to assure the existence of a positive solution to the problem for sufficiently small $\lambda>0$. Our results extend the corresponding results established in the $p$-Laplacian case to the $p(x)$-Laplacian case.
\end{abstract}

2010 Mathematics Subject Classification. 35J70.

1. Introduction. In this paper, we consider the existence of positive solutions for the following $p(x)$-Laplacian-Dirichlet problem of the form

$$
\begin{cases}-\operatorname{div}\left(|\nabla u|^{p(x)-2} \nabla u\right)+m(x)|u|^{p(x)-2} u=\lambda a(x) f(u) & \text { in } \Omega, \\ u=0 & \text { on } \partial \Omega,\end{cases}
$$

where $\Omega$ is a bounded smooth domain in $\mathbb{R}^{N}, \lambda>0$, the function $a(x)$ is allowed to change sign, $p, m$ and $f$ satisfy the following conditions, respectively:

(P) $p \in C^{0}(\bar{\Omega})$ and $1<p_{-}:=\inf _{x \in \bar{\Omega}} p(x) \leq p_{+}:=\sup _{x \in \bar{\Omega}} p(x)<+\infty$.

$(M) m \in L^{\infty}(\Omega)$ and $m(x) \geq 0$ for $x \in \Omega$.

$(F) f: \mathbb{R} \rightarrow \mathbb{R}$ is continuous and $f(0)>0$.

Problem (1.1) involves the variable exponent $p(\cdot)$. The study of various mathematical problems with variable exponent has received considerable attention in recent years. For a survey of this area see $[4,7,20,28]$, and for the application background see $[\mathbf{2 1}, \mathbf{2 7}]$. The existence and multiplicity of solutions to the $p(x)$ Laplacian equations under various hypotheses were studied by many authors (see e.g. $[3,8,10-12,16,23-26,29,30])$. In this paper, we study the existence of a positive solution to problem (1.1) for sufficiently small $\lambda>0$.

The existence of positive solutions to problem (1.1) when $p(x) \equiv p$ (a constant) was obtained in $[\mathbf{2}, \mathbf{5}, \mathbf{6}, \mathbf{1 7}, \mathbf{1 8}]$. In $[\mathbf{2}, \mathbf{5}, \mathbf{6}, \mathbf{1 7}]$ the case that $p=2$ and $m=0$ was investigated, where in [5] the radially symmetric case was investigated. Hai and $\mathrm{Xu}[\mathbf{1 8}]$ investigated the case that $p \in(1, \infty)$ and $m \geq 0$. In $[2,5,6,17,18]$ the authors gave 
some sufficient conditions on $a(x)$ to assure the existence of a positive solution for small values of $\lambda$. We denote by $S_{p}(a)$ the unique solution of the problem

$$
\begin{cases}-\operatorname{div}\left(|\nabla z|^{p-2} \nabla z\right)+m|z|^{p-2} z=a(x) & \text { in } \Omega \\ z=0 & \text { on } \partial \Omega\end{cases}
$$

for $a \in L^{\infty}(\Omega)$. Then the condition given in $[6,17]$ is

$\left(A_{\bar{\varepsilon}}^{\geq}\right)$there exists $\varepsilon>0$ such that $S_{2}\left(a^{+}-(1+\varepsilon) a^{-}\right) \geq 0$ in $\Omega$, where $a^{+}(x)=$ $\max \{0, a(x)\}$ and $a^{-}(x)=a^{+}(x)-a(x)$.

The condition given in $[\mathbf{2}, \mathbf{1 8}]$ is vector.

$\left(A_{*}\right) S_{p}(a)>0$ in $\Omega$ and $\frac{\partial S_{p}(a)}{\partial v}<0$ on $\partial \Omega$, where $v$ denotes the unit outward normal

The $p(x)$-Laplacian is an extension of the $p$-Laplacian. An essential difference between them is that the $p$-Laplacian operator is $(p-1)$ homogeneous, that is, $\Delta_{p}(\lambda u)=$ $\lambda^{p-1} \Delta_{p} u$ for every $\lambda>0$, but the $p(x)$-Laplacian operator, when $p(x)$ is not a constant, is not homogeneous. Our purpose is to extend the corresponding results established in $[\mathbf{2}, \mathbf{5}, \mathbf{6}, \mathbf{1 7}, \mathbf{1 8}]$ on the $p$-Laplacian problems to the $p(x)$-Laplacian case; however, in this respect we face an essential difficulty due to the inhomogeneity of the $p(x)$-Laplacian operator. It is well known that, in the case that $p(x) \equiv p$ (a constant), if $z$ is a positive solution of (1.2), then, by the ( $p-1)$ homogeneity of the $p$-Laplacian operator, for any $\lambda>0, \lambda^{\frac{1}{p-1}} z$ is exactly a positive solution of the problem

$$
\begin{cases}-\operatorname{div}\left(|\nabla z|^{p-2} \nabla z\right)+m|z|^{p-2} z=\lambda a(x) & \text { in } \Omega, \\ z=0 & \text { on } \partial \Omega .\end{cases}
$$

This fact plays an important role in $[\mathbf{2}, \mathbf{5}, \mathbf{6}, \mathbf{1 7}, \mathbf{1 8}]$. It is a pity that, in the $p(x)$-Laplacian case, such fact does not hold. To see this, in Section 2 we give an example which shows that there are $p(x)$ and $a(x)$ such that the corresponding problem (1.2) with $p=p(x)$ has a positive solution, but for sufficiently small $\lambda>0$, the corresponding problem (1.3) with $p=p(x)$ has no positive solution. Such an example shows that the condition of the same form as $\left(A_{\varepsilon}^{\geq}\right)$or $\left(A_{*}\right)$ is not suitable for the variable exponent problems considered in the present paper. In order to achieve our goal we must find some new conditions which are different from $\left(A_{\varepsilon}^{\geq}\right)$and $\left(A_{*}\right)$ in form, but include $\left(A_{\varepsilon}^{\geq}\right)$and $\left(A_{*}\right)$ as a special case when $p$ is a constant.

In Section 2, we give some preliminaries about the $p(x)$-Laplacian and also give an example as mentioned above. In Section 3, we give some sufficient conditions for the existence of a positive solution to problem (1.1) for sufficiently small $\lambda>0$. Our results are a generalization of the corresponding results established in $[\mathbf{2}, \mathbf{5}, \mathbf{6}, \mathbf{1 7}, \mathbf{1 8}]$ for the $p$-Laplacian case to the $p(x)$-Laplacian case.

2. Preliminaries and example. In this paper, if there is no other explanation, it will always be assumed that $\Omega$ is a bounded smooth domain in $\mathbb{R}^{N}$ and $p$ and $m$ satisfy $(P)$ and $(M)$.

The variable exponent Lebesgue space $L^{p(\cdot)}(\Omega)$ is defined by

$$
L^{p(\cdot)}(\Omega)=\left\{u \mid u: \Omega \rightarrow \mathbb{R} \text { is measurable, } \int_{\Omega}|u|^{p(x)} d x<\infty\right\}
$$


with the norm

$$
|u|_{L^{p(\cdot)}(\Omega)}=|u|_{p(\cdot)}=\inf \left\{\sigma>\left.0\left|\int_{\Omega}\right| \frac{u}{\sigma}\right|^{p(x)} d x \leq 1\right\}
$$

The variable exponent Sobolev space $W^{1, p(\cdot)}(\Omega)$ is defined by

$$
W^{1, p(\cdot)}(\Omega)=\left\{u \in L^{p(\cdot)}(\Omega)|| \nabla u \mid \in L^{p(\cdot)}(\Omega)\right\}
$$

with the norm

$$
\|u\|_{W^{1, p(\cdot)}(\Omega)}=\|u\|_{1, p(\cdot)}=|u|_{p(\cdot)}+|\nabla u|_{p(\cdot)} .
$$

Denote by $W_{0}^{1, p(\cdot)}(\Omega)$ the closure of $C_{0}^{\infty}(\Omega)$ in $W^{1, p(\cdot)}(\Omega) .|\nabla u|_{p(\cdot)}$ is an equivalent norm on $W_{0}^{1, p(\cdot)}(\Omega)$. We refer to $[4,7,14,19,22,28]$ for the elementary properties of the space $W^{1, p(x)}(\Omega)$.

$u \in W_{0}^{1, p(\cdot)}(\Omega)$ is said to be a (weak) solution of (1.1) if

$$
\int_{\Omega}\left(|\nabla u|^{p(x)-2} \nabla u \nabla v+m(x)|u|^{p(x)-2} u v\right) d x=\lambda \int_{\Omega} a(x) f(u) v d x, \forall v \in W_{0}^{1, p(\cdot)}(\Omega) .
$$

Define $T=T_{p(\cdot)}: W_{0}^{1, p(\cdot)}(\Omega) \rightarrow\left(W_{0}^{1, p(\cdot)}(\Omega)\right)^{*}$ by

$$
T(u) v=\int_{\Omega}\left(|\nabla u|^{p(x)-2} \nabla u \nabla v+m(x)|u|^{p(x)-2} u v\right) d x, \forall u, v \in W_{0}^{1, p(\cdot)}(\Omega) .
$$

Proposition 2.1. ([12]) The mapping $T: W_{0}^{1, p(\cdot)}(\Omega) \rightarrow\left(W_{0}^{1, p(\cdot)}(\Omega)\right)^{*}$ is a strictly monotone homeomorphism, and is of type $\left(S_{+}\right)$, namely for any sequence $\left\{u_{n}\right\} \subset W_{0}^{1, p(\cdot)}(\Omega)$ for which $u_{n} \rightarrow u$ in $W_{0}^{1, p(\cdot)}(\Omega)$ and $\overline{\lim }_{n \rightarrow \infty} T\left(u_{n}\right)\left(u_{n}-u\right) \leq 0, u_{n}$ must converge strongly to $u$ in $W_{0}^{1, p(\cdot)}(\Omega)$, where ' $\rightarrow$ ' denotes the weak convergence in $W_{0}^{1, p(\cdot)}(\Omega)$.

Denote by $S=S_{p(\cdot)}$ the inverse mapping of $T$. Then the mapping $S=T^{-1}$ : $\left(W_{0}^{1, p(\cdot)}(\Omega)\right)^{*} \rightarrow W_{0}^{1, p(\cdot)}(\Omega)$ is a strictly monotone homeomorphism. We often view $S$ as the solution operator for the problem

$$
\begin{cases}-\operatorname{div}\left(|\nabla u|^{p(x)-2} \nabla u\right)+m(x)|u|^{p(x)-2} u=h(x) & \text { in } \Omega, \\ u=0 & \text { on } \partial \Omega,\end{cases}
$$

namely, we denote by $S(h)$ the (unique) solution of (2.1), and according to the different ranges of $h$ and $S(h)$, we may have the different understandings of the mapping $S$.

Proposition 2.2. (1) For every $h \in L^{\infty}(\Omega)$, (2.1) has a unique solution $S(h)$ and $S(h) \in L^{\infty}(\Omega)$. 
(2) (Comparison principle) The mapping $S: L^{\infty}(\Omega) \rightarrow L^{\infty}(\Omega)$ is increasing, that is, $S(h) \leq S(g)$ in $\Omega$ if $h \leq g$ in $\Omega$.

(3) The mapping $S: L^{\infty}(\Omega) \rightarrow L^{\infty}(\Omega)$ is bounded, and there is a positive constant $C_{*}$, dependent on $p_{+}, p_{-}, N$ and $|\Omega|$, such that

$$
|S(h)|_{L^{\infty}(\Omega)} \leq C_{*} \max \left\{|h|_{L^{\infty}(\Omega)}^{\frac{1}{p_{+}-1}},|h|_{L^{\infty}(\Omega)}^{\frac{1}{p_{-}-1}}\right\} \text { for all } h \in L^{\infty}(\Omega) .
$$

Proof. For statement (1) see [13], and for statement (2) see [10]. Here we only prove statement (3). First, let us consider the case that $h(x) \equiv M$ (a constant). By [10, Lemma 2.1], there exists a positive constant $C_{*}$, dependent on $p_{+}, p_{-}, N$ and $|\Omega|$, such that

$$
|S(M)|_{L^{\infty}(\Omega)} \leq C_{*} \max \left\{|M|^{\frac{1}{p^{+-1}}},|M|^{\frac{1}{p-1}}\right\} \text { for all } M \in \mathbb{R} .
$$

(Note that Lemma 2.1 in [10] was proved for the case that $m=0$, in fact, the proof of the same result in the case when $m \neq 0$ is similar and the constant $C_{*}$ is independent of $m$ ). Then, for any $h \in L^{\infty}(\Omega)$, statement (3) follows from the above inequality for the constant function $M$ and the comparison principle (2).

$p$ is said to be Hölder continuous on $\bar{\Omega}$ if there exist constants $\alpha \in(0,1)$ and $L>0$ such that $|p(x)-p(y)| \leq L|x-y|^{\alpha}$ for all $x, y \in \bar{\Omega}$. $p$ is said to be Log-Hölder continuous on $\bar{\Omega}$ if there exists a positive constant $L$ such that

$$
|p(x)-p(y)| \leq \frac{L}{-\ln |x-y|} \quad \text { for all } x, y \in \bar{\Omega} \text { with }|x-y| \leq \frac{1}{2} .
$$

It is obvious that Lipschitz continuity $\Longrightarrow$ Hölder continuity $\Longrightarrow$ Log-Hölder continuity.

Proposition 2.3. (1) $([\mathbf{1}, \mathbf{1 0}, \mathbf{1 3}])$ When $p$ is Log-Hölder continuous on $\bar{\Omega}$, for every $h \in L^{\infty}(\Omega), S(h)$ is Hölder continuous on $\bar{\Omega}$, and therefore, the mapping $S: L^{\infty}(\Omega) \rightarrow$ $C^{0}(\bar{\Omega})$ is completely continuous.

(2) $([\mathbf{1}, 9,10])$ When $p$ is Hölder continuous on $\bar{\Omega}$, for every $h \in L^{\infty}(\Omega)$, $S(h) \in C^{1, \alpha}(\bar{\Omega})$, and therefore, the mapping $S: L^{\infty}(\Omega) \rightarrow C^{1}(\bar{\Omega})$ is completely continuous.

Proposition 2.4. ([15]) (A strong maximum principle) Suppose that $p$ is Lipschitz continuous on $\bar{\Omega}, h \in L^{\infty}(\Omega), h(x) \geq 0$ for $x \in \Omega$ and $h(x) \not \equiv 0$ in $\Omega$. Then $S(h) \in C^{1, \alpha}(\bar{\Omega})$, $S(h)(x)>0$ for $x \in \Omega$ and $\frac{\partial S(h)}{\partial v}<0$ on $\partial \Omega$.

Propositions 2.1-2.4 are an extension of the corresponding results established in the case that $p$ is a constant.

An essential difference between the $p(x)$-Laplacian and the $p$-Laplacian is that the $p$-Laplacian is homogeneous but the $p(x)$-Laplacian is inhomogeneous. As mentioned in Section 1, in the case that $p$ is a constant, if for a fixed $h \in L^{\infty}(\Omega)$ there holds $S_{p}(h)(x) \geq 0$ (resp. $\left.S_{p}(h)(x)>0\right)$ for $x \in \Omega$, then for every $\lambda>0$, there holds also $S_{p}(\lambda h)(x) \geq 0$ (resp. $\left.S_{p}(\lambda h)(x)>0\right)$ for $x \in \Omega$. However, this is not the case when $p(\cdot)$ is not a constant. To see this, we give an example as follows. 
EXAMPLE. Let $N=1, \Omega=(-1,1), m=0$,

$$
\begin{gathered}
p(r)= \begin{cases}4, & \text { if }|r| \leq \frac{1}{4}, \\
-8\left(|r|-\frac{1}{2}\right)+2, & \text { if } \frac{1}{4} \leq|r| \leq \frac{1}{2}, \\
2, & \text { if } \frac{1}{2} \leq|r| \leq 1,\end{cases} \\
h_{\varepsilon}(r)= \begin{cases}-\varepsilon, & \text { if }|r| \leq \frac{1}{2}, \\
1 & \text { if } \frac{1}{2}<|r| \leq 1 .\end{cases}
\end{gathered}
$$

where $\varepsilon$ is a small positive number.

For this example we have the following

Proposition 2.5. In the above example, there exists $\varepsilon>0$ sufficiently small such that $S_{p(\cdot)}\left(h_{\varepsilon}\right)>0$ in $\Omega$ and

$$
\inf _{r \in(-1,1)} S_{p(\cdot)}\left(\lambda h_{\varepsilon}\right)(r)<0 \quad \text { for sufficiently small } \lambda>0 .
$$

Proof. By the definition of $p(r), p$ is Lipschitz continuous on $\bar{\Omega}$. Noting that when $\varepsilon=0, h_{0} \geq 0$ and $h_{0} \neq \equiv 0$ in $\Omega$, by Proposition 2.4, $S\left(h_{0}\right) \in C^{1}(\bar{\Omega}), S\left(h_{0}\right)(x)>0$ for $x \in \Omega$ and $\frac{\partial S\left(h_{0}\right)}{\partial v}<0$ on $\partial \Omega$. By 2) of Proposition 2.3, for sufficiently small $\varepsilon>0$, we have $S\left(h_{\varepsilon}\right) \in C^{1}(\bar{\Omega}), S\left(h_{\varepsilon}\right)(x)>0$ for $x \in \Omega$ and $\frac{\partial S\left(h_{\varepsilon}\right)}{\partial v}<0$ on $\partial \Omega$. Now let $\varepsilon \in(0,1)$ be small enough. For any $\lambda>0$, denote $u_{\lambda}=S\left(\lambda h_{\varepsilon}\right)$. Then, since $p(\cdot)$ and $h_{\varepsilon}(\cdot)$ are radially symmetric, $u_{\lambda}$ is radially symmetric and it is the unique solution of the following problem:

$$
\left\{\begin{array}{l}
-\left(\left|u_{\lambda}^{\prime}(r)\right|^{p(r)-2} u_{\lambda}^{\prime}(r)\right)^{\prime}=\lambda h_{\varepsilon}(r) \quad \text { in }(0,1) \\
u_{\lambda}(1)=0, u_{\lambda}^{\prime}(0)=0
\end{array}\right.
$$

Indeed, problem (2.3) has a unique solution $u_{\lambda}(r)$ for $r \in[0,1]$, which is expressed by formula (2.4). Setting $u_{\lambda}(r)=u_{\lambda}(-r)$ for $r \in[-1,0]$, then the function $u_{\lambda}(r), r \in[-1,1]$, is radially symmetric and $u_{\lambda}=S\left(\lambda h_{\varepsilon}\right)$.

Denote $\Phi(r, \xi)=|\xi|^{p(r)-2} \xi$ for $r \in[-1,1]$ and $\xi \in \mathbb{R}$. Then for each $r \in[-1,1]$, $\Phi(r, \cdot): \mathbb{R} \rightarrow \mathbb{R}$ is a homeomorphism. Denote by $\Phi_{r}^{-1}$ the inverse mapping of $\Phi(r, \cdot)$, that is

$$
\Phi_{r}^{-1}(\eta)= \begin{cases}\eta^{\frac{1}{p(r)-1}} & \text { if } \eta \geq 0 \\ -|\eta|^{\frac{1}{p(r)-1}} & \text { if } \eta<0 .\end{cases}
$$

Then we have

$$
u_{\lambda}(r)=\int_{r}^{1} \Phi_{t}^{-1}\left(\int_{0}^{t} \lambda h_{\varepsilon}(s) d s\right) d t \quad \text { for } r \in[0,1] .
$$

From the definition of $h_{\varepsilon}$ we have

$$
\int_{0}^{t} h_{\varepsilon}(s) d s \begin{cases}<0 & \text { if } 0<r<\frac{1}{2}+\frac{1}{2} \varepsilon \\ \geq 0 & \text { if } \frac{1}{2}+\frac{1}{2} \varepsilon \leq r \leq 1\end{cases}
$$


and thus by (2.4),

$$
\begin{aligned}
u_{\lambda}(0) & =\int_{0}^{1} \Phi_{t}^{-1}\left(\int_{0}^{t} \lambda h_{\varepsilon}(s) d s\right) d t \\
& =\int_{0}^{\frac{1}{2}+\frac{1}{2} \varepsilon} \Phi_{t}^{-1}\left(\int_{0}^{t} \lambda h_{\varepsilon}(s) d s\right) d t+\int_{\frac{1}{2}+\frac{1}{2} \varepsilon}^{1} \Phi_{t}^{-1}\left(\int_{0}^{t} \lambda h_{\varepsilon}(s) d s\right) d t \\
& <\int_{0}^{\frac{1}{4}} \Phi_{t}^{-1}\left(\int_{0}^{t} \lambda h_{\varepsilon}(s) d s\right) d t+\lambda \int_{\frac{1}{2}+\frac{1}{2} \varepsilon}^{1}\left(t-\frac{1}{2}-\frac{1}{2} \varepsilon\right) d t \\
& \leq-\int_{0}^{\frac{1}{4}}(\lambda \varepsilon t)^{\frac{1}{3}} d t+\lambda \int_{\frac{1}{2}}^{1}\left(t-\frac{1}{2}\right) d t \\
& =-\frac{3}{4}\left(\frac{1}{4}\right)^{\frac{4}{3}} \varepsilon^{\frac{1}{3}} \lambda^{\frac{1}{3}}+\frac{1}{8} \lambda .
\end{aligned}
$$

This shows that, when $\lambda \leq 6^{\frac{3}{2}}\left(\frac{1}{4}\right)^{2} \varepsilon^{\frac{1}{2}}, u_{\lambda}(0)<0$, that is, (2.2) holds.

3. Existence of positive solutions. Let us continue to use the notations as in Sections 1 and 2.

Let

$$
\begin{array}{ll}
\Gamma_{p(\cdot)}^{\geq}=\left\{h \in L^{\infty}(\Omega) \mid S_{p(\cdot)}(h)(x) \geq 0\right. & \text { for } x \in \Omega\}, \\
\Gamma_{p(\cdot)}^{>}=\left\{h \in L^{\infty}(\Omega) \mid S_{p(\cdot)}(h)(x)>0\right. & \text { for } x \in \Omega\} .
\end{array}
$$

It is clear that when $a=0$, problem (1.1) has only a zero solution, and when $a \geq 0$ and $a(x) \not \equiv 0$ for $x \in \Omega$, using the strong maximum principle, we can easily obtain the existence of a positive solution to (1.1) for small $\lambda>0$. In this section, we assume that $a$ is sign-changed, that is, $a$ satisfies the following condition:

$$
\left(A_{\infty}^{ \pm}\right) \quad a \in L^{\infty}(\Omega), a^{+} \neq 0 \text { and } a^{-} \neq 0
$$

TheOREM 3.1. Let $(P),(M),(F)$ and $\left(A_{\infty}^{ \pm}\right)$hold. Suppose the following condition is satisfied:

$\left(A_{\varepsilon, \delta}^{\geq}\right)\left(\right.$resp. $\left(\left(A_{\varepsilon, \delta}^{>}\right)\right)$There are $\varepsilon>0$ and $\delta>0$ such that

$$
\mu\left(a^{+}-(1+\varepsilon) a^{-}\right) \in \Gamma_{p(\cdot)}^{\geq}\left(\text {resp. } \in \Gamma_{p(\cdot)}^{>}\right) \quad \text { for } \mu \in(0, \delta] .
$$

Then for sufficiently small $\lambda>0$, problem (1.1) has a non-negative (resp. a positive) solution.

Proof. We only consider the case of $\left(A_{\varepsilon, \delta}^{>}\right)$because the proof for the case of $\left(A_{\varepsilon, \delta}^{\geq}\right)$ is similar. Let $\varepsilon$ and $\delta$ be as in condition $\left(A_{\varepsilon, \delta}^{>}\right)$. Define $\widetilde{f}: \mathbb{R} \rightarrow \mathbb{R}$ by

$$
\widetilde{f}(t)= \begin{cases}f(t) & \text { for }|t| \leq 1 \\ f(-1) & \text { for } t<-1 \\ f(1) & \text { for } t>1\end{cases}
$$


Consider the following problem:

$$
\begin{cases}-\operatorname{div}\left(|\nabla u|^{p(x)-2} \nabla u\right)+m(x)|u|^{p(x)-2} u=\lambda a(x) \tilde{f}(u) & \text { in } \Omega, \\ u=0 & \text { on } \partial \Omega .\end{cases}
$$

Define $\widetilde{F}(t)=\int_{0}^{t} \widetilde{f}(s) d s$ for $t \in \mathbb{R}$ and

$$
J_{\lambda}(u)=\int_{\Omega}\left(\frac{1}{p(x)}|\nabla u|^{p(x)}+\frac{m(x)}{p(x)}|u|^{p(x)}-\lambda a(x) \widetilde{F}(u)\right) d x, \forall u \in W_{0}^{1, p(\cdot)}(\Omega) .
$$

Obviously, there exists a positive constant $C$ such that $\tilde{f}(t) \mid \leq C$ for all $t \in \mathbb{R}$, this implies that $|\widetilde{F}(t)| \leq C|t|$ for all $t \in \mathbb{R}$. Noting that $p_{-}>1, m \in L^{\infty}(\Omega), m(x) \geq 0$ and $a \in L^{\infty}(\Omega)$, we can see that, for each $\lambda>0$, the functional $J_{\lambda}: W_{0}^{1, p(\cdot)}(\Omega) \rightarrow$ $\mathbb{R}$ is coercive and sequentially weakly lower semi-continuous, and consequently, $J_{\lambda}$ has a global minimizer $u_{\lambda}$ which is a weak solution of problem (3.1). Noting that $\left|\lambda a(x) \widetilde{f}\left(u_{\lambda}\right)\right|_{L^{\infty}(\Omega)} \rightarrow 0$ as $\lambda \rightarrow 0$, by 3 ) of Proposition 2.2 , we have that $\left|u_{\lambda}\right|_{L^{\infty}(\Omega)} \rightarrow 0$ as $\lambda \rightarrow 0$. Now we assume that $\lambda>0$ is small enough such that $\left|u_{\lambda}\right|_{L^{\infty}(\Omega)} \leq 1$. Then $\widetilde{f}\left(u_{\lambda}\right)=f\left(u_{\lambda}\right)$ and so $u_{\lambda}$ is a solution of problem (1.1). Set $\gamma=\frac{\varepsilon}{2+\varepsilon}$. Since $f$ is continuous at 0 and $f(0)>0$, there is $\rho \in(0,1)$ such that

$$
-f(0) \gamma<f(\xi)-f(0)<f(0) \gamma \quad \text { for }|\xi| \leq \rho .
$$

Take $\lambda_{1}>0$ small enough such that $\left|u_{\lambda}\right|_{L^{\infty}(\Omega)} \leq \rho$ for $\lambda \in\left(0, \lambda_{1}\right]$. Then when $\lambda \in\left(0, \lambda_{1}\right]$,

$$
\begin{aligned}
\lambda a(x) f\left(u_{\lambda}(x)\right) & =\lambda\left(a^{+}(x)-a^{-}(x)\right) f\left(u_{\lambda}(x)\right) \\
& =\lambda a^{+}(x) f\left(u_{\lambda}(x)\right)-\lambda a^{-}(x) f\left(u_{\lambda}(x)\right) \\
& \geq \lambda a^{+}(x) f(0)(1-\gamma)-\lambda a^{-}(x) f(0)(1+\gamma) \\
& =\lambda(1-\gamma) f(0)\left(a^{+}(x)-\frac{1+\gamma}{1-\gamma} a^{-}(x)\right) \\
& =\lambda(1-\gamma) f(0)\left(a^{+}(x)-(1+\varepsilon) a^{-}(x)\right) .
\end{aligned}
$$

Let $\lambda_{2}=\frac{\delta}{(1-\gamma) f(0)}$ and $\lambda_{3}=\min \left\{\lambda_{1}, \lambda_{2}\right\}$. Then when $\lambda \in\left(0, \lambda_{3}\right]$, we have that $\lambda(1-$ $\gamma) f(0) \leq \delta$, and by condition $\left(A_{\varepsilon, \delta}^{>}\right)$,

$$
\lambda(1-\gamma) f(0)\left(a^{+}(x)-(1+\varepsilon) a^{-}(x)\right) \in \Gamma_{p(\cdot)}^{>} .
$$

By (3.2) and the comparison principle, $\lambda a(x) f\left(u_{\lambda}(x)\right) \in \Gamma_{p(\cdot)}^{>}$, which shows that $u_{\lambda}$ is a positive solution of problem (1.1).

REMARK 3.1. In Section 1, we mentioned condition $\left(A_{\varepsilon}^{\geq}\right)$which was used in $[6,17]$ for the case that $p=2$. We may extend it to the variable exponent case. For given variable exponent $p(\cdot)$, we say that $a \in L^{\infty}(\Omega)$ satisfies condition $\left(A_{\bar{\varepsilon}}^{\geq}\right)$(resp. $\left(A_{\varepsilon}^{>}\right)$) if the following condition holds:

$\left(A_{\varepsilon}^{\geq}\right)\left(\operatorname{resp} .\left(A_{\varepsilon}^{>}\right)\right)$there exists $\varepsilon>0$ such that

$$
\left(a^{+}-(1+\varepsilon) a^{-}\right) \in \Gamma_{p(\cdot)}^{\geq}\left(\text {resp. } \in \Gamma_{p(\cdot)}^{>}\right) .
$$

Obviously, condition $\left(A_{\varepsilon}^{>}\right)$implies condition $\left(A_{\varepsilon}^{\geq}\right)$. In the case when $p=2$, from the strong comparison principle (i.e. the strong maximum principle) we may see that 
when $a \in L^{\infty}(\Omega) \backslash\{0\}$ satisfies condition $\left(A_{\varepsilon}^{>}\right)$with some $\varepsilon>0, a$ must satisfy condition $\left(A_{\varepsilon_{1}}^{>}\right)$for every $\varepsilon_{1} \in(0, \varepsilon)$. In other words, when $p=2,\left(A_{\varepsilon}^{>}\right)$and $\left(A_{\varepsilon}^{>}\right)$are essentially equivalent to each other. However, in the case when $p \neq 2$, because of lack of the general strong comparison principle, in general, condition $\left(A_{\bar{\varepsilon}}^{\geq}\right)$does not imply condition $\left(A_{\varepsilon_{1}}^{>}\right)$ for $\varepsilon_{1} \in(0, \varepsilon)$. It is clear that, in the case when $p$ is a constant, $\left(A_{\varepsilon}^{\geq}\right)$and $\left(A_{\varepsilon, \delta}^{\geq}\right)$(resp. $\left(A_{\varepsilon}^{>}\right)$and $\left.\left(A_{\varepsilon, \delta}^{>}\right)\right)$are essentially equivalent to each other. Thus our Theorem 3.1 is an extension of Theorem 2 in [6] and Theorem 1.1 in [17] to the $p(x)$-Laplacian case.

For $h \in L^{\infty}(\Omega)$ and $\varepsilon>0$, define

$$
B_{\infty}(h, \varepsilon)=\left\{g \in L^{\infty}(\Omega)|| g-\left.h\right|_{L^{\infty}(\Omega)}<\varepsilon\right\},
$$

and for $\delta>0$, define

$$
K\left(B_{\infty}(h, \varepsilon), \delta\right)=\left\{\mu g \mid \mu \in(0, \delta] \text { and } g \in B_{\infty}(h, \varepsilon)\right\} .
$$

Corollary 3.1. Let $(P),(M),(F)$ and $\left(A_{\infty}^{ \pm}\right)$hold. Suppose the following condition is satisfied:

$\left(K_{\varepsilon, \delta}^{\geq}\right)\left(\operatorname{resp} .\left(K_{\varepsilon, \delta}^{>}\right)\right)$There are $\varepsilon>0$ and $\delta>0$ such that

$$
K\left(B_{\infty}(a, \varepsilon), \delta\right) \subset \Gamma_{p(\cdot)}^{\geq}\left(\text {resp. } \subset \Gamma_{p(\cdot)}^{>}\right) .
$$

Then a satisfies $\left(A_{\varepsilon_{1}, \delta}^{\geq}\right)\left(\right.$resp. $\left.\left(A_{\varepsilon_{1}, \delta}^{>}\right)\right)$for some $\varepsilon_{1}>0$, and consequently, for sufficiently small $\lambda>0$, problem (1.1) has a non-negative (resp. a positive) solution.

Proof. Let $a$ satisfy $\left(K_{\varepsilon, \delta}^{\geq}\right)\left(\right.$resp. $\left.\left(K_{\varepsilon, \delta}^{>}\right)\right)$. Take $\varepsilon_{1} \in\left(0, \frac{\varepsilon}{\mid a^{-} L_{L^{\infty}(\Omega)}}\right)$. Then

$$
\left|\left(a^{+}-\left(1+\varepsilon_{1}\right) a^{-}\right)-a\right|_{L^{\infty}(\Omega)}=\varepsilon_{1}\left|a^{-}\right|_{L^{\infty}(\Omega)}<\varepsilon,
$$

which shows $\left(a^{+}-\left(1+\varepsilon_{1}\right) a^{-}\right) \in B_{\infty}(a, \varepsilon)$. For $\mu \in(0, \delta]$, we have that

$$
\mu\left(a^{+}-\left(1+\varepsilon_{1}\right) a^{-}\right) \in K\left(B_{\infty}(h, \varepsilon), \delta\right) \subset \Gamma_{p(\cdot)}^{\geq}\left(\text {resp. } \subset \Gamma_{p(\cdot)}^{>}\right) .
$$

This shows that $\left(A_{\varepsilon_{1}, \delta}^{\geq}\right)\left(\operatorname{resp} .\left(A_{\varepsilon_{1}, \delta}^{>}\right)\right)$is satisfied, and consequently, by Theorem 3.1, problem (1.1) has a non-negative (resp. a positive) solution for sufficiently small $\lambda>0$.

REMARK 3.2. Let $p \in(1, \infty)$ be a constant and $a \in L^{\infty}(\Omega)$ satisfy condition $\left(A_{*}\right)$, that is $S_{p}(a)>0$ in $\Omega$ and $\frac{\partial S_{p}(a)}{\partial v}<0$ on $\partial \Omega$. Since $S_{p}: L^{\infty}(\Omega) \rightarrow C^{1}(\bar{\Omega})$ is continuous, there exists $\varepsilon>0$ such that $B_{\infty}(a, \varepsilon) \subset \Gamma_{p}^{>}$. In this case, for any $\delta>0, K\left(B_{\infty}(a, \varepsilon), \delta\right) \subset$ $\Gamma_{p}^{>}$holds. This shows that, when $p$ is a constant, condition $\left(A_{*}\right)$ implies condition $\left(K_{\varepsilon, \delta}^{>}\right)$ for some $\varepsilon>0$ and any $\delta>0$. Hence Theorem 1 of Hai and $\mathrm{Xu}[\mathbf{1 8}]$ is a special case of Corollary 3.1.

Now let us consider the radially symmetric case. Suppose that the following condition is satisfied.

$(R) \Omega=B\left(0, r_{0}\right) \subset \mathbb{R}^{N}$ is a ball, $p(x)=p(|x|)=p(r)$ and $a(x)=a(|x|)=a(r)$ are radially symmetric, and $m=0$. 
In this case, the solution of (1.1) is just the solution of the following problem:

$$
\left\{\begin{array}{l}
-\left(r^{N-1}\left|u^{\prime}(r)\right|^{p(r)-2} u^{\prime}(r)\right)^{\prime}=\lambda r^{N-1} a(r) f(u) \quad \text { in }\left(0, r_{0}\right) \\
u\left(r_{0}\right)=0, \quad u^{\prime}(0)=0
\end{array}\right.
$$

Corollary 3.2. Let $(P),(M),(F),\left(A_{\infty}^{ \pm}\right)$and $(R)$ hold. Suppose that a satisfies the following condition

$\left(I_{\tau}\right)$ there exists $\tau>0$ such that

$$
\int_{0}^{s} t^{N-1} a^{+}(t) d t \geq(1+\tau) \int_{0}^{s} t^{N-1} a^{-}(t) d t \quad \text { for } s \in\left(0, r_{0}\right] .
$$

Then a satisfies condition $\left(A_{\varepsilon, \delta}^{>}\right)$with $\varepsilon=\frac{\tau}{2}$ and any $\delta>0$, and consequently, for sufficiently small $\lambda>0$, problem (1.1) has a positive solution. Then

Proof. Put $\varepsilon=\frac{\tau}{2}$. Let any $\mu>0$ be given. Denote $u=S_{p(\cdot)}\left(\mu\left(a^{+}-(1+\varepsilon) a^{-}\right)\right)$.

$$
\left\{\begin{array}{l}
-\left(r^{N-1}\left|u^{\prime}(r)\right|^{p(r)-2} u^{\prime}(r)\right)^{\prime}=\mu r^{N-1}\left(a^{+}-(1+\varepsilon) a^{-}\right) \text {in }\left(0, r_{0}\right), \\
u\left(r_{0}\right)=0, \quad u^{\prime}(0)=0 .
\end{array}\right.
$$

Thus we have, for $r \in\left(0, r_{0}\right]$,

$$
\begin{aligned}
-\left(r^{N-1}\left|u^{\prime}(r)\right|^{p(r)-2} u^{\prime}(r)\right) & =\mu \int_{0}^{r} t^{N-1}\left(a^{+}(t)-\left(1+\frac{\tau}{2}\right) a^{-}(t)\right) d t \\
& \geq \frac{\mu \tau}{2} \int_{0}^{r} t^{N-1} a^{-}(t) d t \geq 0 .
\end{aligned}
$$

This shows that $u^{\prime}(r) \leq 0$ for all $r \in\left(0, r_{0}\right)$. Noting that $\int_{0}^{r_{0}} t^{N-1} a^{-}(t) d t>0$, we have $u^{\prime}\left(r_{0}\right)<0$, and therefore $u(r)>0$ for $r \in\left[0, r_{0}\right)$ because $u\left(r_{0}\right)=0$. This proves that $\mu\left(a^{+}-(1+\varepsilon) a^{-}\right) \in \Gamma_{p(\cdot)}^{>}$for any $\mu>0$, that is, condition $\left(A_{\varepsilon, \delta}^{>}\right)$with $\varepsilon=\frac{\tau}{2}$ and any $\delta>0$ is satisfied, and consequently, by Theorem 3.1, problem (1.1) has a positive solution for sufficiently small $\lambda>0$.

Remark 3.3. Condition $\left(I_{\tau}\right)$ was proposed by Các, Fink and Gatica [5] for the case that $p=2$. Note that condition $\left(I_{\tau}\right)$ used in this paper is the same as in [5], and it is independent of $p(\cdot)$. The verification of condition $\left(I_{\tau}\right)$ is often easy, for example, it is easy to see that, in the radially symmetric case, the function $a$, defined by

$$
a(r)= \begin{cases}1 & \text { if }|r| \leq \frac{r_{0}}{2}, \\ -\varepsilon & \text { if } \frac{r_{0}}{2}<|r| \leq r_{0},\end{cases}
$$

where $\varepsilon \in\left(0, \frac{1}{2^{N}-1}\right)$, satisfies condition $\left(I_{\tau}\right)$ with $\tau \in\left(0, \frac{1}{\varepsilon\left(2^{N}-1\right)}-1\right)$. Of course, as was mentioned in $[\mathbf{2}, \mathbf{6}],\left(I_{\tau}\right)$ is a stronger condition to assure the existence of a positive solution to problem (3.4) for small values of $\lambda$.

REMARK 3.4. Let $\Omega, m, p(\cdot)$ and $a=h_{\varepsilon}$ be as in the example given in Section 2, where $\varepsilon>0$ is sufficiently small, and let $f(t)=1$ for all $t \in \mathbb{R}$. Proposition 2.5 shows 
that, in this case, condition $\left(A_{*}\right)$ as well as condition $\left(A_{\varepsilon_{1}}^{>}\right)$with small $\varepsilon_{1}>0$ is satisfied but the corresponding problem (1.1) has no positive solution for sufficiently small $\lambda>0$.

Finally, we give an example in which the condition $\left(A_{\varepsilon, \delta}^{>}\right)$put in Theorem 3.1 is satisfied but the condition $\left(I_{\tau}\right)$ put in Corollary 3.2 is not satisfied.

EXAMPLE 3.5. Let $N=1, \Omega=(-1,1), m=0$,

$$
\begin{aligned}
& p(r)= \begin{cases}2, & \text { if }|r| \leq \frac{1}{2}, \\
8\left(|r|-\frac{1}{2}\right)+2, & \text { if } \frac{1}{2} \leq|r| \leq \frac{3}{4}, \\
4, & \text { if } \frac{3}{4} \leq|r| \leq 1,\end{cases} \\
& a(r)= \begin{cases}-\frac{1}{8}, & \text { if }|r| \leq \frac{1}{2}, \\
1 & \text { if } \frac{1}{2}<|r| \leq 1 .\end{cases}
\end{aligned}
$$

Take $\varepsilon=1$. we will show that there exists $\delta>0$ such that condition $\left(A_{1, \delta}^{>}\right)$is satisfied, that is, $\mu\left(a^{+}-(1+1) a^{-}\right) \in \Gamma_{p(\cdot)}^{>}$for $\mu \in(0, \delta]$. Denote $u_{\mu}=S_{p(\cdot)}\left(\mu\left(a^{+}-2 a^{-}\right)\right)$. Then $u_{\lambda}$ is radially symmetric and it is the unique solution of the following problem:

$$
\left\{\begin{array}{l}
-\left(\left|u_{\mu}^{\prime}(r)\right|^{p(r)-2} u_{\mu}^{\prime}(r)\right)^{\prime}=\mu\left(a^{+}-2 a^{-}\right)(r) \quad \text { in }(0,1), \\
u_{\mu}(1)=0, u_{\mu}^{\prime}(0)=0 .
\end{array}\right.
$$

Thus, we have

$$
u_{\mu}^{\prime}(r)=-\Phi_{r}^{-1}\left(\int_{0}^{r} \mu\left(a^{+}(s)-2 a^{-}(s)\right) d s\right) \quad \text { for } r \in(0,1) .
$$

It is sufficient to prove that $u_{\mu}(r)>0$ for sufficiently small $\mu>0$ and all $r \in[0,1)$. We may assume $\mu \in(0,1)$. Noting that when $r \in\left(0, \frac{1}{2}\right]$,

$$
\int_{0}^{r} \mu\left(a^{+}(s)-2 a^{-}(s)\right) d s=\int_{0}^{r}-\frac{1}{4} \mu d s=-\frac{1}{4} \mu r<0,
$$

and when $r \in\left(\frac{1}{2}, 1\right)$,

$$
\begin{aligned}
\int_{0}^{r} \mu\left(a^{+}(s)-2 a^{-}(s)\right) d s & =\int_{0}^{\frac{1}{2}}-\frac{1}{4} \mu d s+\int_{\frac{1}{2}}^{r} \mu d s \\
& =-\frac{1}{8} \mu+\left(r-\frac{1}{2}\right) \mu=\left(r-\frac{5}{8}\right) \mu,
\end{aligned}
$$

we can see that $u_{\mu}^{\prime}(r)>0$ for $r \in\left(0, \frac{5}{8}\right), u_{\mu}^{\prime}(r)<0$ for $r \in\left(\frac{5}{8}, 1\right)$, and $u_{\mu}$ attains its maximum at $r=\frac{5}{8}$. Since $u_{\mu}(1)=0$, we have that $u_{\mu}(r)>0$ for $r \in\left[\frac{5}{8}, 1\right)$ and

$$
\begin{aligned}
u_{\mu}\left(\frac{5}{8}\right) & >u_{\mu}\left(\frac{3}{4}\right)=-\int_{\frac{3}{4}}^{1} u_{\mu}^{\prime}(r) d r=\int_{\frac{3}{4}}^{1} \Phi_{r}^{-1}\left(\left(r-\frac{5}{8}\right) \mu\right) d r \\
& \geq \int_{\frac{3}{4}}^{1}\left(\frac{1}{8} \mu\right)^{\frac{1}{4-1}} d r=\frac{1}{4} \cdot \frac{1}{2} \mu^{\frac{1}{3}} .
\end{aligned}
$$


For $r \in\left[0, \frac{5}{8}\right)$ we have

$$
\begin{aligned}
u_{\mu}(r) & \geq u_{\mu}(0)=u_{\mu}\left(\frac{5}{8}\right)-\int_{0}^{\frac{5}{8}} u_{\mu}^{\prime}(r) d r \geq u_{\mu}\left(\frac{5}{8}\right)-\int_{0}^{\frac{5}{8}} \Phi_{r}^{-1}\left(\frac{1}{4} \mu\right) d r \\
& \geq u_{\mu}\left(\frac{5}{8}\right)-\int_{0}^{\frac{5}{8}}\left(\frac{1}{4} \mu\right)^{\frac{1}{p\left(\frac{5}{8}\right)-1}} d r \\
& =\frac{1}{8} \mu^{\frac{1}{3}}-\frac{5}{8} \cdot\left(\frac{1}{4} \mu\right)^{\frac{1}{2}} .
\end{aligned}
$$

It follows that when $\mu \in\left(0,\left(\frac{2}{5}\right)^{6}\right), u_{\mu}(r)>0$ for all $r \in[0,1)$. This shows that the condition $\left(A_{1, \delta}^{>}\right)$is satisfied for $\delta \in\left(0,\left(\frac{2}{5}\right)^{6}\right)$. It is obvious that the condition $\left(I_{\tau}\right)$ is not satisfied because for any $\tau>0$ and $s \in\left(0, \frac{1}{2}\right)$,

$$
0=\int_{0}^{s} a^{+}(t) d t<(1+\tau) \int_{0}^{s} a^{-}(t) d t .
$$

ACKNOWLEDGEMENTS. This research was supported by National Natural Science Foundation of China $(10671084,10971087)$. The author is grateful to the reviewer for valuable comments.

\section{REFERENCES}

1. E. Acerbi and G. Mingione, Regularity results for a class of functionals with nonstandard growth, Arch. Ration. Mech. Anal. 156 (2001), 121-140.

2. G. A. Afrouzi and K. J. Brown, Positive solutions for a semilinear elliptic problem with sign-changing nonlinearity, Nonlinear Anal. 36 (1999), 507-510.

3. C. O. Alves and M. A. S. Souto, Existence of solutions for a class of problems in $\mathbb{R}^{N}$ involving the $p(x)$-Laplacian, Prog. Nonlinear Differ. Equ. Appl. 66 (2005), 17-32.

4. S. Antontsev and S. Shmarev, Elliptic equations with anisotropic nonlinearity and nonstandard conditions, in Handbook of differential equations, stationary partial differential equations, vol. 3 (Chipot M. and Quittner P., Editors) (Elsevier B. V., North Holland, Amsterdam, 2006), 1-100.

5. N. P. Các, A. M. Fink and J. A. Gatica, Nonnegative solutions to the radial Laplacian with nonlinearity that changes sign, Proc. Amer. Math. Soc. 123 (1995), 1393-1398.

6. N. P. Các, J. A. Gatica and Y. Li, Positive solutions to semilinear problems with coefficient that changes sign, Nonlinear Anal. 37 (1999), 501-510.

7. L. Diening, P. Hästö and A. Nekvinda, Open problems in variable exponent Lebesgue and Sobolev spaces, in FSDONA04 proceedings (Drábek P. and Rákosník J. Editors), The Conference held in Milovy, May 28-June 2, 2004, (Math. Inst. Acad. Sci. Czech Republic, Praha, 2005), 38-58.

8. T.-L. Dinu, On a nonlinear eigenvalue problem in Sobolev spaces with variable exponent, Sib. Elektron. Mat. Izv. 2 (2005), 208-217.

9. X. L. Fan, Global $C^{1, \alpha}$ regularity for variable exponent elliptic equations in divergence form, J. Differ. Equ. 235 (2007), 397-417.

10. X. L. Fan, On the sub-supersolution method for $p(x)$-Laplacian equations, J. Math. Anal. Appl. 330 (2007), 665-682.

11. X. L. Fan, Remarks on eigenvalue problems involving the $p(x)$-Laplacian, J. Math. Anal. Appl. 352 (2009), 85-98.

12. X. L. Fan and Q. H. Zhang, Existence of solutions for $p(x)$-Laplacian Dirichlet problems, Nonlinear Anal. 52 (2003), 1843-1852. 
13. X. L. Fan and D. Zhao, A class of De Giorgi type and Hölder continuity, Nonlinear Anal. 36 (1999), 295-318.

14. X. L. Fan and D. Zhao, On the spaces $L^{p(x)}(\Omega)$ and $W^{k, p(x)}(\Omega)$, J. Math. Anal. Appl. 263 (2001), 424- 446.

15. X. L. Fan, Y. Z. Zhao, Q. H. Zhang, A strong maximum principle for $p(x)$-Laplace equations, Chin. Ann. Math. Ser. A 24 (2003) 495-500 (in Chinese), English translation: Chin. J. Contemp. Math. 24 (2003), 277-282.

16. Y. Q. Fu and $\mathrm{X}$. Zhang, A multiplicity result for $p(x)$-Laplacian problem in $\mathbb{R}^{N}$, Nonlinear Anal. 70 (2009), 2261-2269.

17. D. D. Hai, Positive solutions to a class of elliptic boundary value problems, J. Math. Anal. Appl. 227 (1998), 195-199.

18. D. D. Hai and $\mathrm{X}$. $\mathrm{Xu}$, On a class of quasilinear problems with sign-changing nonlinearities, Nonlinear Anal. 64 (2006), 1977-1983.

19. P. Harjulehto and P. Hästö, An overview of variable exponent Lebesgue and Sobolev spaces, in Future Trends in Geometric Function Theory (Herron D., Editor) (RNC Workshop, Jyväskylä, 2003), 85-93.

20. P. Harjulehto, P. Hästö, U. V. Lê and M. Nuortio, Overview of differential equations with non-standard growth, Nonlinear Anal. 72 (2010), 4551-4574.

21. V. V. Jikov, S. M. Kozlov and O. A. Oleinik, Homogenization of differential operators and integral functional (Springer-Verlag, Berlin, 1994). Translated from the Russian by G. A. Yosifan.

22. O. Kováčik and J. Rákosnik, On spaces $L^{p(x)}$ and $W^{k, p(x)}$, Czech. Math. J. 41(116) (1991), $592-618$.

23. P. Marcellini, Regularity and existence of solutions of elliptic equations with $(p, q)$ growth conditions, J. Differ. Equ. 90 (1991), 1-30.

24. M. Mihăilescu and V. Rădulescu, A multiplicity result for a nonlinear degenerate problem arising in the theory of electrorheological fluids, Proc. R. Soc. Lond. Ser. A 462 (2006), 2625-2641.

25. M. Mihăilescu and V. Rădulescu, On a nonhomogeneous quasilinear eigenvalue problem in Sobolev spaces with variable exponent, Proc. Amer. Math. Soc. 135 (2007), 29292937.

26. M. Mihăilescu and V. Rădulescu, Continuous spectrum for a class of nonhomogeneous differential operators, Manuscr. Math. 125 (2008), 157-167.

27. M. Růžička, Electrorheological fluids: Modeling and mathematical theory (SpringerVerlag, Berlin, 2000).

28. S. Samko, On a progress in the theory of Lebesgue spaces with variable exponent: maximal and singular operators, Integral Transforms Spec. Funct. 16 (2005), 461-482.

29. Q. H. Zhang, Existence and asymptotic behavior of positive solutions for variable exponent elliptic systems, Nonlinear Anal. 70 (2009), 305-316.

30. V. V. Zhikov, On some variational problems, Russ. J. Math. Phys. 5 (1997), 105-116. 